\title{
European Respiratory Society guideline on long-term management of children with bronchopulmonary dysplasia
}

\author{
Liesbeth Duijts (1) $^{1,2}$, Evelien R. van Meel ${ }^{1}$, Laura Moschino ${ }^{3}$, Eugenio Baraldi ${ }^{3}$, \\ Magda Barnhoorn ${ }^{4}$, Wichor M. Bramer ${ }^{5}$, Charlotte E. Bolton (106), Jeanette Boyd ${ }^{7}$, \\ Frederik Buchvald ${ }^{8}$, Maria Jesus del Cerro ${ }^{9}$, Andrew A. Colin ${ }^{10}$, Refika Ersu ${ }^{11,12}$, \\ Anne Greenough ${ }^{13}$, Christiaan Gremmen ${ }^{4}$, Thomas Halvorsen (10 ${ }^{14,15}$, \\ Juliette Kamphuis ${ }^{7}$, Sailesh Kotecha ${ }^{16}$, Kathleen Rooney-Otero ${ }^{17}$, Sven Schulzke ${ }^{18}$, \\ Andrew Wilson ${ }^{19}$, David Rigau ${ }^{20}$, Rebecca L. Morgan ${ }^{21}$, Thomy Tonia ${ }^{22}$, \\ Charles C. Roehr ${ }^{23,24}$ and Marielle W. Pijnenburg ${ }^{1}$
}

@ERSpublications

This guideline provides recommendations on monitoring and treatment of children with established bronchopulmonary dyplasia older than 36 weeks postmenstrual age or after discharge from the hospital, based on PICO questions relevant for clinical care http://bit.ly/2m9CfKt

Cite this article as: Duijts L, van Meel ER, Moschino L, et al. European Respiratory Society guideline on long-term management of children with bronchopulmonary dysplasia. Eur Respir J 2020; 55: 1900788 [https://doi.org/10.1183/13993003.00788-2019].

ABSTRACT This document provides recommendations for monitoring and treatment of children in whom bronchopulmonary dysplasia (BPD) has been established and who have been discharged from the hospital, or who were $>36$ weeks of postmenstrual age. The guideline was based on predefined Population, Intervention, Comparison and Outcomes (PICO) questions relevant for clinical care, a systematic review of the literature and assessment of the evidence using the Grading of Recommendations, Assessment, Development and Evaluation (GRADE) approach. After considering the balance of desirable (benefits) and undesirable (burden, adverse effects) consequences of the intervention, the certainty of the evidence, and values, the task force made conditional recommendations for monitoring and treatment of BPD based on very low to low quality of evidence. We suggest monitoring with lung imaging using ionising radiation in a subgroup only, for example severe BPD or recurrent hospitalisations, and monitoring with lung function in all children. We suggest to give individual advice to parents regarding daycare attendance. With regards to treatment, we suggest the use of bronchodilators in a subgroup only, for example asthma-like symptoms, or reversibility in lung function; no treatment with inhaled or systemic corticosteroids; natural weaning of diuretics by the relative decrease in dose with increasing weight gain if diuretics are started in the neonatal period; and treatment with supplemental oxygen with a saturation target range of 90-95\%. A multidisciplinary approach for children with established severe BPD after the neonatal period into adulthood is preferable. These recommendations should be considered until new and urgently needed evidence becomes available.

This article has supplementary material available from erj.ersjournals.com

This document was endorsed by the ERS Executive Committee on September 10, 2019.

Received: 18 April 2019 | Accepted after revision: 30 Aug 2019

Copyright OERS 2020 


\section{Introduction}

Bronchopulmonary dysplasia (BPD), also called chronic lung disease of prematurity, is a chronic respiratory disease that predominantly affects children born preterm. Advanced perinatal care has improved the survival of children born extremely preterm; however, the incidence of BPD has not decreased [1]. Improved survival is mainly due to the introduction of antenatal management, including maternal corticosteroid administration, intratracheal surfactant administration, less aggressive mechanical ventilation strategies and targeted oxygen therapy, which consequently have led to a different form of BPD [2-26]. Since 1999, BPD is defined as oxygen need for $\geqslant 28$ days from birth until 36 weeks of postmenstrual age (PMA) $[27,28]$. While in earlier years BPD was associated with aggressive mechanical ventilation, improved ventilation changed the histological phenotype of BPD from a predominantly post-traumatic condition leading to the formation of hyaline membranes (old form of BPD), to one where pulmonary changes are characterised by a global alveolar development arrest (new form of BPD) [27, 29]. The precise maturational trajectory of airways, lungs and related vessels in extreme preterm, early extra-uterine life is not fully known, but is likely to comprise airway-, lung- and vascular-driven pathology leading to severe chronic respiratory and vascular diseases across the life course, and potentially shortens life expectancy. Once discharged from the neonatal unit, children with BPD are at a high risk of rehospitalisation due to higher susceptibility to viral infections, decreased nutritional state or poorer neurological outcome, leading to increased healthcare utilisation and costs [30]. Apart from advising on preventative measures, the application of supportive measures is paramount. Therefore, physicians and caretakers need to assess the disease progression and tailor treatment adequately. In addition, studies have shown that children with BPD have an impaired lung structure, lower lung function, including declining lung function over time, and increased risk of respiratory symptoms in later life [31-41]. This suggests that BPD partly reflects an ongoing chronic respiratory disease with long-term consequences and not just stabilised structural lung damage after the neonatal period. BPD in childhood may form a new group of chronic obstructive pulmonary disease (COPD) in adulthood.

Although several tools have been studied for their utility in monitoring children with BPD, to date there are no guidelines on comprehensive monitoring strategies for children in whom BPD has been established and who are discharged from the hospital $[42,43]$. Furthermore, most studies have so far largely focused on preventing, rather than treating established BPD. Interventional studies beyond the neonatal period, such as use of inhaled or systemic corticosteroids, bronchodilators and long-term oxygen treatment are scarce. A clear consensus and recommendations with grading evidence on how to monitor and treat children with BPD at the long term is lacking.

Therefore, we undertook a systematic review of the literature and developed recommendations following the Grading of Recommendations Assessment, Development and Evaluation (GRADE) [44], to inform decisions regarding the monitoring and treatment of children with BPD. Specifically, we focused on those children with the new form of BPD in whom BPD already had been established and were discharged from the hospital, or who were older than 36 weeks of postmenstrual age. This population is meant when referring to children with BPD throughout this manuscript. The target audience of this guideline includes

Affiliations: ${ }^{1}$ Dept of Pediatrics, Division of Respiratory Medicine and Allergology, Erasmus MC, University Medical Center Rotterdam, Rotterdam, The Netherlands. ${ }^{2}$ Dept of Pediatrics, Division of Neonatology, Erasmus MC, University Medical Center Rotterdam, Rotterdam, The Netherlands. ${ }^{3}$ Dept of Women's and Children's Health, University of Padua, Padua, Italy. ${ }^{4}$ Lung Foundation Netherlands, Amersfoort, The Netherlands. ${ }^{5}$ Medical Library, Erasmus MC, University Medical Center Rotterdam, Rotterdam, The Netherlands. ${ }^{6}$ NIHR Nottingham BRC Respiratory Theme and Division of Respiratory Medicine, University of Nottingham, Nottingham, UK. ${ }^{7}$ European Lung Foundation (ELF), Sheffield, UK. ${ }^{8}$ Pediatric Pulmonary Service, DBLC, Rigshospitalet, Copenhagen, Denmark. ${ }^{9}$ Pediatric Cardiology, Ramón y Cajal University Hospital, Madrid, Spain. ${ }^{10}$ Division of Pediatric Pulmonology, Miller School of Medicine, University of Miami, Miami, FL, USA. ${ }^{11}$ Division of Respirology, Marmara University Istanbul, Istanbul, Turkey. ${ }^{12}$ Division of Respirology, University of Ottowa, Children's Hospital of Eastern Ontario, Ottawa, ON, Canada. ${ }^{13}$ Women and Children's Health, School of Life Course Sciences, Faculty of Life Sciences and Medicine, King's College London, London, UK. ${ }^{14}$ Dept of Pediatrics, Haukeland University Hospital, Bergen, Norway. ${ }^{15}$ Dept of Clinical Science, University of Bergen, Bergen, Norway. ${ }^{16}$ Dept of Child Health, School of Medicine, Cardiff University, Cardiff, UK. ${ }^{17}$ Division of Hospital Medicine, Nemours Children's Hospital, Orlando, FL, USA. ${ }^{18}$ Dept of Neonatology, University Children's Hospital Basel UKBB, Basel, Switzerland. ${ }^{19}$ Dept of Respiratory and Sleep Medicine, Princess Margaret Hospital for Children, Perth, Australia. ${ }^{20}$ Iberoamerican Cochrane Centre, Barcelona, Spain. ${ }^{21}$ Dept of Health Research Methods, Evidence and Impact, McMaster University, Hamilton, ON, Canada. ${ }^{22}$ Insitute of Social and Preventive Medicine, University of Bern, Bern, Switzerland. ${ }^{23}$ Dept of Paediatrics, Medical Sciences Division, University of Oxford, Oxford, UK. ${ }^{24}$ Newborn Services, John Radcliffe Hospital, Oxford University Hospitals, Oxford, UK.

Correspondence: Liesbeth Duijts, Erasmus MC, University Medical Center Rotterdam, Sp-3435; PO Box 2060, 3000 CB Rotterdam, The Netherlands. Email: I.duijtsderasmusmc.nl 
specialists in respiratory medicine, paediatricians and/or neonatologists who manage children with BPD. Other healthcare physicians such as respiratory nurses and policy makers may also benefit from this guideline. This guideline provides the basis for rational decisions in the monitoring and treatment of specifically children in whom BPD has been established and who have been discharged from the hospital, or were $>36$ weeks PMA. Clinicians, patients and their parents/care-givers, third-party payers, stakeholders or the courts should never view the recommendations contained in these guidelines as mandatory. Although evidence-based guidelines can summarise the best available evidence regarding the effects of an intervention in a given patient population, they cannot take into account all of the unique clinical circumstances that may arise when managing an individual patient.

\section{Methods}

\section{Task force composition}

The chairs applied for a task force on BPD, which was approved and funded by the European Respiratory Society (ERS). The task force consisted of a multidisciplinary group of clinicians, scientific researchers, methodologists or patient representatives with expertise in the paediatric respiratory $(n=11)$, neonatology $(n=3)$, paediatric cardiology $(n=1)$, adult respiratory $(n=1)$, epidemiology $(n=3)$ and patient involvement $(n=4)$ fields related to long-term monitoring and treatment of children with BPD. All representatives had intensive experience in long-term follow-up of children born preterm or with BPD. Task force members were from Europe, the United States and Australia. Two junior members/trainees of the ERS, a parent of a patient with BPD, an adult patient with BPD and national (Lung Foundation Netherlands) and international (European Lung Foundation) patient representatives were active members of the committee. In addition, methodologists from the ERS provided expertise in guideline development following the GRADE approach. Potential conflicts of interest were disclosed and managed according to ERS policies.

\section{Formulation of the topics and questions}

Task force members compiled a list of topics that they considered important and relevant to the monitoring and treatment of children with established BPD after the neonatal period. Discussion among the task force members was applied to identify the eight most relevant and important questions to be addressed in this guideline. Questions related to the topics were phrased using the Population, Intervention, Comparison and Outcomes (PICO) format. The population (P) consisted specifically of children with the new form of BPD, in whom BPD had been established and who were discharged from the hospital or were older than 36 weeks PMA. The interventions (I) comprised monitoring with lung imaging or lung function, discouraging daycare attendance, and treatment with inhaled bronchodilators, inhaled corticosteroids, systemic corticosteroids, diuretics or oxygen. The comparison (C) group were those without the intervention. Specific important and critical outcomes $(\mathrm{O})$ defined for each question are presented in table 1 . The eight questions for children with BPD were:

1. Does monitoring with lung imaging versus no lung imaging; and

2. Does monitoring with lung function versus no lung function; and

3. Does discouraging daycare attendance versus not discouraging daycare attendance; and

4. Does treatment with inhaled bronchodilators versus no inhaled bronchodilators; and

5. Does treatment with inhaled corticosteroids versus no inhaled corticosteroids; and

6. Does treatment with systemic corticosteroids versus no systemic corticosteroids; and

7. Does treatment with diuretics versus no diuretics; and

8. Does treatment with oxygen versus no oxygen affect outcomes which are defined as important or critical?

Thereafter, the questions "Does treatment with inhaled corticosteroids versus no inhaled corticosteroids"; and "Does treatment with systemic corticosteroids versus no inhaled corticosteroids affect important and critical defined outcomes" were combined for practical reasons. For each question, a PICO working group was composed with a leader and between two and four members.

\section{Rating the importance of outcomes}

The task force identified BPD morbidity and related outcomes after discharge or after 36 weeks of PMA that they considered relevant to each question. These comprised number and severity of respiratory symptoms, adverse growth, hospital admissions, abnormalities on computed tomography (CT), reduced physical exercise capacity, pulmonary hypertension, use of inhaled bronchodilators, use of inhaled corticosteroids, use of systemic corticosteroids, use of diuretics, prolonged duration of supplemental oxygen need, side-effects, adverse neurodevelopment, decreased quality of life, mortality or impaired lung function, depending on each question under study. Task force members rated the importance of each outcome a priori, using a scale from 1 to 9. A rating of 1-3 was assigned to outcomes of low importance, 4-6 to important outcomes and 7-9 to outcomes critical for decision-making. Individual ratings were 
TABLE 1 Outcomes defined as "important" or "critical" for decision-making for each Population, Intervention, Comparison and Outcomes (PICO) question related to the intervention for children with bronchopulmonary dysplasia

Intervention Outcome

Monitoring with lung imaging

Monitoring with lung function

Discourage daycare attendance

Treatment with inhaled bronchodilators

Treatment with inhaled/systemic corticosteroids

Treatment with diuretics

Treatment with oxygen
Number and severity of respiratory symptoms, adverse growth, hospital admissions, reduced physical exercise capacity, prolonged duration of supplemental oxygen need, side-effects, adverse neurodevelopment, decreased quality of life, mortality or impaired lung function

Number and severity of respiratory symptoms, hospital admissions, CT abnormalities, reduced physical exercise capacity, use of inhaled bronchodilators, use of inhaled corticosteroids, use of systemic corticosteroids, prolonged duration of supplemental oxygen need, adverse neurodevelopment, quality of life or mortality

Number and severity of respiratory symptoms, adverse growth, hospital admissions, prolonged duration of supplemental oxygen need, adverse neurodevelopment, quality of life or mortality

Number and severity of respiratory symptoms, hospital admissions, reduced physical exercise capacity, prolonged duration of supplemental oxygen need, quality of life, mortality or impaired lung function

Number and severity of respiratory symptoms, adverse growth, hospital admissions, reduced physical exercise capacity, pulmonary hypertension, prolonged duration of supplemental oxygen need, side-effects, adverse neurodevelopment, quality of life, mortality or impaired lung function

Number and severity of respiratory symptoms, hospital admissions, pulmonary hypertension, prolonged duration of supplemental oxygen need, side-effects, adverse neurodevelopment, quality of life, mortality or impaired lung function

Number and severity of respiratory symptoms, adverse growth, hospital admissions, reduced physical exercise capacity, pulmonary hypertension, prolonged duration of supplemental oxygen need, side-effects, adverse neurodevelopment, quality of life, mortality or impaired lung function

CT: computed tomography.

summarised and evaluated by the co-chairs and an ERS methodologist. A final rating was proposed to all task force members, and approved. All outcomes were categorised as "not important", "important" or "critical" for decision-making (supplementary table S1). Only "important" or "critical" outcomes were used in the literature search and decision-making [45].

\section{Literature search methods}

We conducted literature searches in Embase, MEDLINE Ovid, Cochrane Central Registry of Trials and Web of Science Core Collection until July 11, 2018 (last data search). We included meta-analyses and systematic reviews of randomised trials, randomised trials and retrospective or prospective cohort studies published from 1999, in which BPD was defined. Detailed scripts of the search terms created by a librarian (WB) are given in the methods part of the supplementary material and supplementary table S1. Scripts were broadly set to limit missing of relevant articles. Conference abstracts were omitted.

\section{Study selection}

For each PICO question, working groups selected relevant articles in three stages, by title screening and abstract screening; full article screening; and reading full articles to summarise findings related to the PICO item. A minimum of two group members were required to independently review the relevant articles to minimise potential bias. Inclusion criteria were the study population of children in whom BPD had been established and who were discharged from the hospital, or were $>36$ weeks PMA, and studying the specifically defined monitoring and treatment tools in relation to the defined outcomes of interest. Exclusion criteria were incorrect population under study (no children, children with old BPD definition or born preterm or with very low birthweight without specific diagnosis of BPD as opposed to children with BPD only as second-best), incorrect monitoring or treatment intervention, study focused on prevention instead of monitoring or treatment of BPD, outcomes of interest not reported, no abstract or full text available or no English text available. When no articles for the PICO directly fulfilled these criteria, indirect articles that indirectly fulfilled these criteria were included using a less favourable study design, preterm-born children (as opposed to children with BPD) or the old form of BPD (as opposed to the new form of BPD). A discussion was held between the independent PICO working group members when no consensus was reached to include or exclude identified articles. A physician-epidemiologist (LD), one junior ERS member-epidemiologist (EM) and one junior member-trainee (LM) read the identified articles, and discussed these to reach consensus on the selection of final articles if differences in opinion to include or exclude the articles were present. When no indirect articles for the PICO category were found, experience of local, regional or national management of task force members was asked, summarised and discussed if relevant. 


\section{Evidence synthesis and grading}

With guidance from the ERS methodologists, relevant data were extracted from the selected studies for each PICO item taking the outcomes into account that were rated "important" or "critical" for decision-making. We graded the effect estimates for the body of the evidence for each outcome to determine our certainty in the evidence, and presented the findings using the GRADEpro Guideline Development Tool (http://gdt.gradepro.org/app/). We primarily used findings from only one type of study design, preferably observational studies for monitoring questions and randomised controlled trials (RCTs) for treatment questions, to create a summary of evidence, and where appropriate used findings of other types of study designs to complement recommendations. Data were not amenable to pooling.

\section{Formulating recommendations}

The evidence profiles were sent to the task force members for review. Using an iterative consensus process conducted face-to-face and via email, recommendations were formulated on the basis of the balance of desirable (benefits) and undesirable (burden, adverse effect) consequences of the intervention, the certainty of the evidence, values, balance of effects, required resources, costs, equity, acceptability and feasibility, using the evidence-to-decision framework [46]. Whether all domains could be assessed depended on the availability of the evidence per PICO. Recommendations could either be strong or conditional (weak) (table 2). A strong recommendation for an intervention was made when the task force was confident that the desirable effects outweighed the undesirable effects, while a strong recommendation against an intervention was made when the task force was confident that the undesirable effects outweighed the desirable effects. A conditional recommendation for an intervention was made if the task force concluded that the desirable effects probably outweighed the undesirable effects, but was not confident, while a conditional recommendation against the intervention was made if the task force concluded that the undesirable effects probably outweighed the desirable effects, but was not confident.

Reasons for making a conditional recommendation included low or very low certainty in the quality of evidence, a close balance between the desirable and undesirable consequences, or underlying values and preferences, equity, acceptability or feasibility in the direction opposite to that of the desirable effects (e.g. the desirable consequences of an intervention clearly outweigh the undesirable consequences taking into account that in some healthcare systems or situations the intervention is not widely acceptable or feasible to implement).

\section{Manuscript preparation}

The initial draft of the manuscript was prepared by a physician-epidemiologist (LD) and a junior ERS member-epidemiologist (EM) and reviewed by a methodologist. Thereafter, both the manuscript and the supplementary material were reviewed, edited and approved by all task force members prior to submission.

\section{Results}

The results of the evidence assessment are presented in supplementary tables S3.1-3.4. A summary of the recommendations is presented in table 3. For each question, the number of potentially relevant papers ranged from 197 to 4329, and the number of final papers from four to none. When formulating the recommendations, required resources, costs, equity, acceptability and feasibility were not taken into account due to lack of evidence.

TABLE 2 Interpretation of the strength of the recommendations [95]

Implications Strong recommendation Conditional (weak) recommendation

For patients

For policy-makers

For clinicians
Most individuals in this situation would want the recommended course of action and only a small proportion would not. Formal decision aids are not likely to be needed to help individuals make decisions consistent with their values and preferences.

Most individuals should receive the intervention. Adherence to this recommendation according to the guideline could be used as a quality criterion or performance indicator.

\footnotetext{
The recommendation can be adapted as policy in most
} situations.
The majority of individuals in this situation would want the suggested course of action, but many would not.

Recognise that different choices will be appropriate for individual patients and that clinicians must help each patient arrive at a management decision consistent with his or her values and preferences. Decision aids may be useful helping individuals making decisions consistent with their values and preferences.

Policy-making will require substantial debate and involvement of various stakeholders. 


\section{Review of the evidence addressing the question on lung imaging \\ PICO 1}

In children with BPD, does monitoring with lung imaging versus no lung imaging affect important and critical defined outcomes?

\section{Summary of the evidence}

No direct evidence that would answer this question in an appropriate way was identified. Indirect evidence was provided by four studies that examined the relationship of lung imaging with lung function or duration of supplemental oxygen need (supplementary table S3.1) [47-50]. 21 school-children with BPD (mild $\mathrm{n}=9$, moderate $\mathrm{n}=4$, severe $\mathrm{n}=8$ ) were offered the opportunity to undergo high-resolution CT (HRCT) scans [47]. The rate of severe BPD was higher compared to those not participating in scanning. Mean (range) age of the children was 12.7 (8.7-16.7) years. Higher HRCT scores were related to lower forced expiratory volume in $1 \mathrm{~s}\left(\mathrm{FEV}_{1}\right)(\beta-4.23,95 \% \mathrm{CI}-6.97--1.49 ; \mathrm{p}=0.004)$ and maximal mid-expiratory flow $(\beta-3.45,95 \% \mathrm{CI}-6.10--0.80 ; \mathrm{p}=0.013)$, but not to gas exchange as measured by diffusing capacity of the lung for carbon monoxide. A retrospective study among 19 children with BPD observed that all children at a median (range) age of 14.6 (1.5-53.7) months had CT abnormalities, which were not associated with clinical outcomes such as gestational age, type and duration of mechanical ventilation and BPD severity [48]. In a retrospective review, 41 very low birthweight infants with BPD, who had exacerbations in the past 6 months at a mean age underwent HRCT scans and lung function tests at a mean age of 16 months. Maximal expiratory flow at functional residual capacity $\left(V_{\operatorname{maxFRC}}\right)$ and functional residual capacity (FRC) were measured by the squeeze technique [50]. An increased number of triangular subpleural opacities and of limited linear opacities on CT were associated with a lower FRC ( $\mathrm{r}$

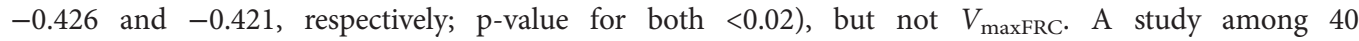
preterm-born children (median (range) age 27 (24-32) weeks observed that those remaining oxygen dependent at a post-conception age of 36 weeks had significantly higher chest radiograph scores at 1 month (median 9 months, range 7-20 months) than those not chronically oxygen dependent (median 3 months, range $0-13$ months $)(\mathrm{p}<0.05)$ [49].

\section{Certainty of the evidence}

The certainty of the evidence was considered very low.

\section{Strength of the recommendation}

Conditional for the intervention.

\section{Task force recommendation}

The task force suggests lung imaging to monitor children with BPD in subgroups only, for example children with severe $\mathrm{BPD}$, severe respiratory symptoms and/or recurrent hospital admissions due to respiratory morbidity (conditional recommendation based on very low certainty of evidence).

TABLE 3 Recommendations for the monitoring and treatment of children with bronchopulmonary dysplasia (BPD)

\section{Question}

Recommendation

Strength

Quality of evidence

\begin{tabular}{|c|c|c|c|}
\hline 1 & $\begin{array}{l}\text { We suggest monitoring with lung imaging using ionising radiation in a subgroup only le.g. children } \\
\text { with severe course of BPD, severe respiratory symptoms, and/or recurrent hospital admissions due } \\
\text { to respiratory morbidityl }\end{array}$ & Conditional & Very low \\
\hline 2 & We suggest monitoring with lung function & Conditional & Very low \\
\hline 4 & $\begin{array}{l}\text { We suggest that treatment with bronchodilators could be optional for subgroups (e.g. children with } \\
\text { severe course of BPD, severe respiratory or asthma-like symptoms, recurrent hospital admission } \\
\text { due to respiratory morbidity, exercise intolerance or reversibility in lung function) }\end{array}$ & Conditional & Very low \\
\hline 8 & We suggest supplemental oxygen with saturation target range of $90-95 \%$ & Conditional & Very low \\
\hline
\end{tabular}

Details of the recommendations are provided in the main text. 
Justification of the recommendation

Among the presented studies with indirect evidence to use lung imaging as a monitoring tool, the study population consisted of children defined with the old form of BPD [51]; the studies used retrospective or cross-sectional data collection with potential risk of bias; or not enough numerical data were reported to be able to judge imprecision. Therefore, the evidence was considered very low. In clinical practice, task force members agreed that given the low certainty of evidence and potential side-effects of radiation, monitoring with lung imaging would be justified only in subgroup of children with severe BPD, severe respiratory symptoms, recurrent hospitalisations or equivalent. For example, a chest CT with intravenous contrast could be considered to exclude other diagnoses, which may affect treatment strategies.

\section{Other considerations}

Almost all children with established BPD seem to have lung structure abnormalities measured by lung imaging [50, 52]. However, studies are mostly among children from an outpatient clinic, and proper control groups are often lacking. In addition, the natural course of lung structural abnormalities and of normal alveolarisation in early life is not fully known.

\section{Suggestions for future research}

Recently, nonionising magnetic resonance imaging (MRI) scan protocols for children with BPD have been developed, and a quiet-breathing MRI scan independently assessed structural abnormalities of BPD and disease severity and predicted short-term outcomes at discharge from the neonatal intensive care unit [53, 54]. This technique is a promising monitoring tool for long-term outcomes. Further studies are warranted to examine the predictive value of lung imaging, preferably nonradiant, on long-term outcomes of children with established BPD. Studies using lung imaging such as CT or MRI in the neonatal phase might be considered to better define the severity of BPD, or to diagnose or exclude other causes of BPD.

\section{Review of the evidence addressing the question on lung function}

PICO 2

In children with BPD, does monitoring with lung function versus no lung function affect important and critical defined outcomes?

\section{Summary of the evidence}

No direct evidence that would answer this question in an appropriate way was identified. Indirect evidence was provided by two studies among preterm-born children. The first study showed that among children born extremely preterm, the ratio of tidal expiratory flow at $50 \%$ of expired volume $\left(\mathrm{TEF}_{50}\right)$ to peak tidal expiratory flow (PTEF), which reflects airway obstruction, measured by electromagnetic inductance plethysmography, predicts respiratory morbidity in the first year of life (area under curve $0.723,95 \%$ CI 0.55-0.86) (supplementary table S3.2) [55]. In addition, $\mathrm{TEF}_{50} / \mathrm{PTEF}$ was lower in the group with respiratory morbidity in the first year of life than in the group without $(73.5$ versus 79.9 , $\mathrm{p}$-value $=0.03$ ) Other tidal breathing lung function measures did not differ between those with and without respiratory morbidity. Another prospective cohort among 163 preterm-born children measured tidal breathing and performed multiple breath washout measurements during sleep at the age of 44 weeks PMA [56]. After adjustment for confounders, a higher respiratory rate and higher tidal volume were associated with a decreased and increased risk of wheeze, respectively, in the first year of life (OR 0.69, 95\% CI 0.50-0.96 and OR 1.40, 95\% CI 1.04-1.90), respectively), and a higher time to peak tidal expiratory flow to expiratory time ratio with less bronchodilator inhalation therapy during the first year of life (OR 0.56, 95\% CI 0.35-0.89). Other lung function measures, such as FRC and lung clearance index were not associated with wheeze, inhalation therapy or rehospitalisation, and none of the lung function measures were associated with home oxygen therapy. The additional value of lung function tests was tested by adding them to prediction models for wheezing in the first year of life based on BPD classification, the clinical risk index for babies score, or clinical standard predictors such as sex, PMA and days of mechanical ventilation. However, adding lung function to either of the three models did not improve prediction of wheeze (area under the curve of model with versus without added lung function (likelihood ratio test $\mathrm{p}$-value) 0.63 versus $0.54(\mathrm{p}=0.15), 0.62$ versus $0.52(\mathrm{p}=0.08)$ and 0.71 versus $0.68(\mathrm{p}=0.12))$.

\section{Certainty of the evidence}

The certainty of the evidence was considered very low. 
Task force recommendation

The task force suggests using lung function to monitor children with BPD (conditional recommendation based on very low certainty of evidence).

\section{Justification of the recommendation}

No studies have been performed that examined the potential beneficial effect of lung function monitoring on important and critical defined outcomes in children with BPD. Among the studies with indirect evidence to use lung function as a monitoring tool, the study population consisted of preterm-born children, not specifically children with $\mathrm{BPD}$, or potential confounders were not taken into account. Therefore, the evidence was considered very low. No evidence was found that monitoring children with BPD with lung function reduces morbidity and related outcomes. However, for clinical practice, task force members agreed that monitoring using lung function would be justified despite the lack of evidence. Lung function, specifically spirometry and related bronchodilator response at older ages, is an objective measure, is associated with lung function in adulthood, and with increased risks of morbidity and mortality, has sex-, age-, height- and ethnicity-adjusted reference ranges and has no potential side-effects. Furthermore, lung function could act as a potential indicator for the risk of lung and related vascular diseases in adulthood.

\section{Other considerations}

Many prospective and retrospective cohort studies have examined lung function at later ages among children with BPD $[37,57,58]$, compared with preterm-born children without BPD $[37,57,58]$ or term-born children [37]. A recent meta-analysis of $>50$ studies showed that children who were born preterm and were diagnosed with BPD had a $16 \%$ lower $\mathrm{FEV}_{1}$, compared with children born at term [32]. Similarly, a review of 18 studies showed that in those with BPD, compared with children born at term at age 6-19 years, $\mathrm{FEV}_{1}$ was consistently lower [34]. However, a large heterogeneity in results was observed, suggesting variation in expression of the disease or differences in populations studied, with mostly diagnoses of the old form of BPD. Furthermore, children born preterm without BPD or born preterm across the full gestational age range have a lower lung function at later age [32, 59], which suggests altered airway and lung maturation or mediation by specific ventilation strategies [60]. Children with BPD often respond less to bronchodilators and have a lower fractional exhaled nitric oxide, a measure of eosinophilic airway inflammation, compared with children with asthma [61, 62]. This suggests that airway reactivity through eosinophilic airway inflammation is probably not involved in BPD. Previous studies observed elevated neutrophils and oxidative stress in airways, measured by induced sputum and exhaled breath condensate respectively, in children aged 11 years or adolescents born preterm compared with children born at term $[63,64]$. This suggests that BPD reflects an ongoing respiratory disease after birth with long-term consequences and not just stabilised structural lung damage after the neonatal period. The possible adverse effects of BPD on lung clearance index, a measure of ventilation heterogeneity of the lungs and a well-accepted and applicable lung function test for children aged $<5$ years, and on exercise capacity, are not fully clear [65-68]. Some differences in lung function in early life have shown to be persistent in adulthood [69], which suggests that the expected optimal peak in lung function development is not reached. The relation of lung function measures with lung structure and risk of respiratory morbidity in children with BPD is not fully clear.

Spirometry seems the most useful method for longitudinal follow-up of lung growth and airway obstruction in school-age children with BPD. For preschool children (age $\leqslant 4$ years) with BPD, the forced oscillation technique and multiple breath washout tests are the most applicable regarding technique and validity $[43,70]$. However, reported studies have small sample sizes and limitations, and the success rate in routine clinical practice without sedation is considered low.

\section{Suggestions for future research}

Further studies, observational or RCT, are warranted to examine the predictive value of lung function on long-term lung structure and respiratory morbidity of children with established BPD, and its value in monitoring responses to treatment.

\section{Review of the evidence addressing the question on daycare attendance \\ PICO 3}

In children with $\mathrm{BPD}$, does discouraging daycare attendance versus not discouraging daycare attendance affect important and critical defined outcomes? 
Certainty of the evidence

The certainty of the evidence was considered very low.

Strength of the recommendation

Conditional for either the intervention or the comparison.

\section{Task force recommendation}

The task force suggests giving individual advice to parents regarding daycare attendance for children with BPD (conditional recommendation based on very low certainty of evidence).

\section{Justification of the recommendation}

Due to the lack of evidence, all task force members were asked what they would advise in their own clinical practice. Most do not discourage daycare attendance; nor do they encourage daycare attendance. The younger the child and the potentially higher prevalence of infectious diseases in specific seasons of the year, the more reluctant the task force members were to encourage daycare attendance. The first winter in young, severely affected children with established BPD would be of most concern for attending daycare. It was mentioned that daycare attendance has positive effects, e.g. on social development. The task force group noted that parental leave regulations differ greatly among countries, for example 12 months in Scandinavian countries versus 3 months in the Netherlands, which could influence the decision to take the child to daycare.

\section{Other considerations}

Advice should be based on local experience, age of the child, season of the year and parental wishes and possibilities.

\section{Suggestions for future research}

Studies are needed to examine the effects of daycare attendance on number and severity of respiratory symptoms, adverse growth, hospital admissions, duration of supplemental oxygen need, neurodevelopment and quality of life.

\section{Review of the evidence addressing the question on inhaled bronchodilators}

$\mathrm{PICO} 4$

In children with BPD, does treatment with inhaled bronchodilators versus no inhaled bronchodilators affect important and critical defined outcomes?

Summary of the evidence

This could not be given because no articles were available.

Certainty of the evidence

The certainty of the evidence was considered very low.

Strength of the recommendation

Conditional recommendation for the intervention.

\section{Task force recommendation}

The task force suggests treatment with bronchodilators for children with BPD in subgroups only, for example children with severe BPD, those with asthma-like symptoms, recurrent hospital admission due to respiratory morbidity, exercise intolerance or reversibility in lung function (conditional recommendation based on very low certainty of evidence).

\section{Justification of the recommendation}

Due to the lack of evidence, a discussion among the group members of the task forces was held on the use of inhaled bronchodilators in practice. It was suggested that treatment with inhaled bronchodilators is optional for subgroups only, e.g. for children with severe BPD, those with asthma-like symptoms, recurrent hospital admission due to respiratory morbidity, exercise intolerance or bronchodilator reversibility in lung function. Potential benefits for some of these children have been experienced by task force group members. Some clinical practices start bronchodilators as a trial, and only continue if significant improvement in respiratory symptoms, lung function, if available, or number of hospitalisations or emergency visits is shown. 
Other considerations

Effects of treatment with inhaled bronchodilators should be carefully monitored by symptoms or lung function if applicable, or reduction of number of hospitalisations or emergency visits before chronically applied.

\section{Suggestions for future research}

Most studies have focused on treatment with bronchodilators of children at $<36$ weeks PMA [71]. Further studies are urgently needed to examine the use of inhaled bronchodilators in children with BPD.

\section{Review of the evidence addressing the question on the use of inhaled/systemic corticosteroids} PICO 5/6

In children in with BPD, does treatment with inhaled or systemic corticosteroids versus no inhaled or systemic corticosteroids affect important and critical defined outcomes?

\section{Summary of the evidence}

No direct evidence that would answer this question in an appropriate way was identified. Indirect evidence was obtained from one study, a crossover RCT that recruited 18 children born preterm (mean gestational age 28 weeks) at the age of 10.5 months, who had symptoms that were not controlled despite regular use of bronchodilators [72] (supplementary table S3.3). Children received either $200 \mu \mathrm{g}$ of beclomethasone dipropionate or placebo twice daily for two 6-week periods, separated by a 2-week washout period. During the active period, as compared to the placebo period, respiratory symptoms decreased (37\% improvement in symptom score, p-value <0.001). During the active period, FRC increased significantly (30 versus $36 \mathrm{~mL} \cdot \mathrm{kg}^{-1}, \mathrm{p}<0.002$ ), while there was no change in FRC during the placebo treatment period (31 versus $\left.32 \mathrm{~mL} \cdot \mathrm{kg}^{-1}\right)$.

Certainty of the evidence

The certainty of the evidence was low.

Strength of the recommendation

Conditional against the intervention.

\section{Task force recommendation}

The task force suggests not to treat with inhaled or systemic corticosteroids for children with BPD (conditional recommendation based on low certainty of evidence). If the treating physician considers the use of inhaled/systemic corticosteroids of additional value, for example children with severe BPD, severe respiratory symptoms, recurrent hospitalisations or equivalent, and not controlled with regular use of bronchodilators, the effects of treatment with inhaled/systemic corticosteroids should be carefully monitored during a trial period before being applied chronically.

\section{Justification of the recommendation}

Only one study was available, which was not performed specifically in children with the new form of BPD, but in preterm-born children who had uncontrolled respiratory symptoms. Additionally, loss to follow-up was a methodological concern. Lastly, the use of corticosteroids may have side-effects and/or can lead to adverse events, which need to be outweighed by the possible benefits. Therefore, the evidence was considered low. Since the use of corticosteroids may lead to side-effects, the task force did not deem it justified to recommend treatment with inhaled corticosteroids.

\section{Other considerations}

Other evidence is provided by a cohort of 63 preterm-born children, followed for 4 months, with a median age of 10 years at the time of the study [73]. Those with bronchial obstruction, increased responsiveness to inhaled bronchodilators and/or abnormal diurnal peak expiratory flow variation were included. In total, 18 children met these criteria and received inhaled budesonide $0.8 \mathrm{mg} \cdot \mathrm{m}^{-2}$ per day in two doses for the first month, followed by $0.4 \mathrm{mg} \cdot \mathrm{m}^{-2}$ per day in two doses for another 3 months. Lung function was measured by spirometry twice daily at home, and at the clinic at baseline, after 1 month and after 4 months of budesonide treatment. Additionally, children kept a record of any respiratory symptoms, defined as cough or wheezing. Budesonide treatment did not lead to any changes in lung function measures at the clinic, although there was a decrease in diurnal peak expiratory flow variation at home both after 1 and 4 months of budesonide treatment. The symptom score after 1 month of budesonide, but not after 4 months, was significantly lower. When the task force discussed the recommendations, it was taken into account that the indirect evidence for the use of corticosteroids was not strong, that there is a difference in using corticosteroids as standard treatment, or at the time of uncontrolled disease, and that corticosteroids 
should be used with caution, especially since the effects on preterm-born, still-growing lungs are not yet known. If the treating physician considers the use of inhaled/systemic corticosteroids of additional value, for example children with severe $\mathrm{BPD}$, severe respiratory symptoms, recurrent hospitalisations or equivalent, and not controlled with regular use of bronchodilators, the effects of treatment with inhaled/ systemic corticosteroids should be carefully monitored during a trial period before being chronically applied. Monitoring could be by number and severity of symptoms, improvement of lung function, if applicable, or by number of hospitalisations or emergency visits.

\section{Suggestions for future research}

Further studies are urgently needed to examine the use of inhaled or systemic corticosteroids in children with BPD.

\section{Review of the evidence directly addressing the question on diuretics}

PICO 7

In children in with BPD, does treatment with diuretics versus no diuretics affect important and critical defined outcomes?

\section{Summary of the evidence}

No direct evidence was identified that would answer this question in an appropriate way. Indirect evidence was provided by one study [74] (supplementary table S3.4). An RCT showed that, among infants with oxygen-dependent BPD who were clinically stable, there were no differences in number of rehospitalisations for respiratory deterioration (diuretic group 22 rehospitalisations in 14 of the patients versus placebo group 19 rehospitalisations in six of the patients), pulmonary function tests, or total duration of supplemental oxygen use (diuretic group $133 \pm 53$ days versus placebo group $147 \pm 71$ days) and no differences in side-effects including nephrocalcinosis, supplemental electrolytes or hearing deficits between the groups after 36 weeks of PMA. Only FRC measured between 9 weeks after weaned from oxygen and diuretics and 1 year of corrected gestational age was increased in the diuretics group. At 1 year of corrected gestational age, the FRC/thoracic gas volume had improved in both the diuretic group $(0.89 \pm 0.18)$ and the placebo group $(0.97 \pm 0.11)$.

\section{Certainty of the evidence}

The certainty of the evidence was considered very low.

\section{Strength of the recommendation}

Conditional for either the intervention or the comparison.

\section{Task force recommendation}

For those children with BPD who already received treatment with diuretics from the neonatal phase or neonatal intensive care unit onwards, the task force suggests natural weaning by the relative decrease in dose with increasing weight gain (conditional recommendation based on very low certainty of evidence). If the treating physician considers the use of diuretics of additional value, for example when clinical signs of fluid retention are present, the effects of treatment with diuretics should be carefully monitored during a trial period before chronically applied.

\section{Justification of the recommendation}

No intervention studies examined the potential beneficial effect of diuretics on important and critical defined outcomes in children in whom BPD has been established and were discharged from the hospital, or who were older than 36 weeks PMA. In the presented study, the exact method of randomisation was unclear, potential confounders were not taken into account, the intention to treat analysis was not fully clear, and additional furosemide supplementation differed between the groups (diuretic group none out of 22 patients versus placebo group five out of 21 patients, $\mathrm{p}<0.05$ ). The study group did not contain children with the new form of BPD. Therefore, the evidence was considered very low.

Other considerations

None.

Further studies are needed to examine the use of diuretics in children with BPD. 
Review of the evidence directly addressing the question on oxygen

PICO 8

In children with BPD, does treatment with oxygen versus no oxygen affect important and critical defined outcomes?

Summary of the evidence

This could not be provided because no articles were available.

Certainty of the evidence

The certainty of the evidence was considered very low.

Strength of the recommendation

Conditional recommendation for the intervention

\section{Task force recommendation}

The task force suggests for children with BPD that supplemental oxygen with a minimum saturation target level of $90 \%$ should be maintained until further studies are performed (conditional recommendation based on very low certainty of evidence).

\section{Justification of the recommendation}

In a study among children born at $<30$ weeks of gestation who were still dependent on supplemental oxygen at 32 weeks of gestation were randomised into target saturation range 91-94\% versus 95-98\% [75]. This study found no difference in growth (weight, length, head circumference at 38 weeks PMA or corrected age of 12 months, or weight and length <10th percentile, or head circumference below the third percentile), rehospitalisation rate, retinopathy of prematurity stage 3 or 4, major developmental abnormality (blindness, cerebral palsy or general quotient on revised Griffiths Mental Developmental Scale $<2$ SD below mean), psychosocial measures or death. Children with higher target saturation did have longer duration of oxygen use, compared to children with lower target saturation. A review of the literature concluded that no studies showed a conclusive proof of the optimal target saturation in post-term oxygen therapy in children born preterm on several outcomes [76]. Saturation targets used were not uniform across different studies. The suggestion was that saturation levels $<90 \%$ should be avoided, and that levels $>92-94 \%$ might be protective against adverse effects. We consider a minimum threshold oxygen saturation measured by pulse oximetry $\left(S_{\mathrm{pO}_{2}}\right)$ of $90 \%$, not lower, since a recent review of the literature found no studies that showed a conclusive proof of the optimal target saturation in post-term oxygen therapy in children born preterm for beneficial effects on several health outcomes [76]. Our suggestion of using a cut-off $S_{\mathrm{pO}_{2}}$ value of $90 \%$ for considering home oxygen is based on low-grade evidence, as is the minimum threshold of $93 \%$ as stated in a recent American Thoracic Society (ATS) guideline [77]. A cut-off value of $90 \% \mathrm{~S}_{\mathrm{pO}_{2}}$ instead of $93 \% \mathrm{~S}_{\mathrm{pO}_{2}}$ will require fewer infants to be discharged on home oxygen therapy, which alleviates a relative financial burden of healthcare system resources without running an unduly high risk of compromising patient outcomes. However, we do emphasise on the need for future studies to define the optimal saturation targets for children in whom BPD already has been established and are discharged from the hospital, or who were older than 36 weeks PMA, taking into account potential effects on nonpulmonary outcomes.

\section{Other considerations}

The BOOST II trial, an international RCT comparing an oxygen saturation target of $85-89 \%$ with $91-95 \%$ for children born before 28 weeks of gestation, demonstrated that a saturation target $<90 \%$ was associated with an increased risk of death before discharge [78]. This finding led to an early stop of the trial. Results of studies comparing different saturation targets on health outcomes after discharge are lacking. The British Thoracic Society guideline on supplementary home oxygen found C and D levels of evidence (of A to $\mathrm{D}$ categories) for supplementary oxygen in children with chronic neonatal lung disease to reduce or prevent pulmonary hypertension, reduce intermittent desaturations, reduce airway resistance, promote growth and neurodevelopment, and to possibly reduce associated risk of sudden unexplained death in infancy [79]. Home oxygen treatment should be recommended, as oxygen at home is preferable to a prolonged hospital stay for both quality of life and psychological impact for the infant, parents and family, and as it saves days in hospital due to earlier discharge despite a significant readmission rate. Specific saturation targets are not provided. The ATS guideline on home oxygen therapy for children recommends home oxygen therapy for patients with BPD complicated by chronic hypoxaemia, based on very low-quality evidence [77]. Home oxygen therapy seemed to increase growth rate; short-term oxygen use decreases mean pulmonary artery pressure; and nocturnal oxygen use improved sleep duration and decreased arousal. The utility of home monitoring on informing temporary decline of BPD is not fully known. 
Suggestions for future research

Further studies are urgently needed to examine the optimal saturation targets of oxygen use in children with BPD.

\section{General considerations}

The new form of BPD is characterised predominantly by an arrest in development of airways and lungs, specifically alveoli, pulmonary vascular development, and to a lesser extent by iatrogenic lung damage [27]. Clinically, the new form of BPD is defined as oxygen need for $\geqslant 28$ days from birth until 36 weeks PMA [27]. However, this definition of BPD is currently under debate as the pathological process of BPD is a sliding scale, its features may also be present in preterm born children without BPD, and it does not seem to allow prediction of outcomes. In the future, potentially biomarkers in blood and exhaled breath, lung function in early life, clinical parameters and quiet-breathing MRI scan may all help to better define BPD and predict short-term outcomes, and additionally long-term outcomes and treatment responses dependent upon or independent of each other [53]. As a consequence of the focus on identifying monitoring and treatment strategies for the current ERS guideline, not every new and promising avenue of monitoring or treatment could ultimately be pursued. It may therefore require future updates to integrate new emerging monitoring and treatment strategies, as for example long-term monitoring and treatment of pulmonary hypertension in children with BPD, which is suggested to be an under-diagnosed condition [80-83]. Paediatric cardiologists should be more intensively involved in multidisciplinary follow-up of children with BPD from discharge into adulthood. Future research should not be limited to children with $\mathrm{BPD}$, but should also include children born across the full range of gestational ages.

For children born $<32$ weeks of gestational age, BPD severity is based on the amount of oxygen needed at 36 weeks PMA, or discharge to home $[28,84]$. An objective, reliable and safe test for BPD severity is the "oxygen reduction test", a standardised assessment of oxygen saturation during a timed stepwise reduction of administered oxygen to room air [85]. Objectively determining the severity of BPD may be important for identifying children most at risk for later lung, pulmonary vascular or other sequelae, implying that more close monitoring and treatment might be needed.

In addition to the considered and rated outcomes, extremely preterm born children are at increased risks of adverse ophthalmologic and renal outcomes. The development or deterioration of retinopathy of prematurity in our defined population was considered very small, but cannot be excluded for late-onset retinopathy. Therefore, retinopathy of prematurity warrants close monitoring specifically when discussing saturation limits if supplemental oxygen is needed. Future studies are warranted to examine interventions related to monitoring and treatment of adverse renal outcomes [86]. Additionally, the incidence of chronic pulmonary vascular disease in children and adults born preterm in the new BPD era are not fully clear. Results from studies among children born within the old and new BPD eras suggest that they have an increased risk of subclinical pulmonary hypertensive vascular disease, exercise induced pulmonary hypertension, right ventricular dysfunction and autonomic dysfunction [82, 87-90]. Protocols for screening and diagnosing these adverse health outcomes, specifically pulmonary hypertensive vascular diseases, have been suggested $[81,83,91]$. As with any lung disease or general health, exposure to smoking should be strongly discouraged and omitted.

In line with this task force, a workgroup among experts held by the National Institute of Child Health and Human Development in the United States concluded that there is an urgent need of studies on postnatal management to decrease the severity of $\mathrm{BPD}$, improve respiratory and medication management of established BPD, including BPD-associated pulmonary hypertension, and to obtain more information on the long-term outcomes of BPD [92]. Prospective, structured, standardised and multidisciplinary follow-up of children with BPD from discharge into adulthood is needed, and may help to generate important data for future monitoring and treatment studies. It depends on the (regions) of countries if neonatologists alone monitor and treat children with BPD for much longer than immediately after discharge. Some countries have set up a multidisciplinary outpatient clinic for children with severe BPD with equally and important involvement of subspecialists, including paediatric-pulmonologists, neonatologists, paediatric-cardiologists, ear-nose-throat physicians, physiotherapists, psychologists and social workers/case managers. Transition of such care systems into adulthood is needed, and research related to these multidisciplinary clinics may lead to new insights and improve long-term outcomes $[93,94]$.

Studies on monitoring or treatment of children with BPD are challenging. BPD is a rare disease, and preterm-born children are a vulnerable group of children, which hampers clinical trials. Also, relevant health outcomes at a young age are difficult to define, and long-term follow-up studies are ideally needed. Similarly, studies on the long-term airway-, lung- and vascular-driven pathophysiology related to gestational age at birth, ventilation and oxygen concentration strategies leading to potential BPD subtypes 
in older children or adults are lacking. According to the task force members, all efforts should be made to design and perform studies in children with BPD to improve quality of life and prevent short- and long-term consequences across the life course.

\section{Summary}

The task force utilised comprehensive syntheses to inform its judgments regarding the balance of desirable (benefits) and undesirable (burden, adverse effects) consequences of the intervention, certainty of the evidence, and values, and made conditional recommendations for all interventions. We suggest monitoring with lung imaging using ionising radiation in a subgroup only, for example severe BPD, severe symptoms, recurrent hospitalisations (conditional recommendation based on very low certainty of evidence) and monitoring with lung function in all children (conditional recommendation based on very low certainty of evidence). The task force suggests to give individual advice to parents regarding daycare attendance (conditional recommendation based on very low certainty of evidence). With regards to treatment, the task force suggests treatment with bronchodilators in a subgroup only, for example children with severe BPD, asthma-like symptoms and/or recurrent hospital admissions due to respiratory morbidity (conditional recommendation based on very low certainty of evidence), while treatment with inhaled or systemic corticosteroids is not suggested (conditional recommendation based on low certainty of evidence). The task force suggests not to start diuretics in children with BPD unless clinical signs of fluid retention are present, and for those children with BPD who already received treatment with diuretics from the neonatal phase or neonatal intensive care unit onwards, the task force suggests natural weaning by the relative decrease in dose with increasing weight gain (conditional recommendation based on very low certainty of evidence). The task force suggests supplemental oxygen with a minimum saturation target of $90 \%$ (conditional recommendation based on very low certainty of evidence). A multidisciplinary approach for children with established severe BPD with involvement of subspecialists from discharge after the neonatal period into adulthood is desirable. These recommendations should be considered until new and urgently needed evidence becomes available.

The guidelines published by the European Respiratory Society (ERS) incorporate data obtained from a comprehensive and systematic literature review of the most recent studies available at the time. Health professionals are encouraged to take the guidelines into account in their clinical practice. However, the recommendations issued by this guideline may not be appropriate for use in all situations. It is the individual responsibility of health professionals to consult other sources of relevant information, to make appropriate and accurate decisions in consideration of each patient's health condition and in consultation with that patient and the patient's caregiver where appropriate and/or necessary, and to verify rules and regulations applicable to drugs and devices at the time of prescription.

Conflict of interest: L. Duijts has nothing to disclose. E.R. van Meel has nothing to disclose. L. Moschino has nothing to disclose. E. Baraldi has nothing to disclose. M. Barnhoorn has nothing to disclose. W.M. Bramer has nothing to disclose. C.E. Bolton has nothing to disclose. J. Boyd is an employee of the European Lung Foundation. F. Buchvald has nothing to disclose. M.J. del Cerro has nothing to disclose. A.A. Colin has nothing to disclose. R. Ersu has nothing to disclose. A. Greenough reports grants and honoraria for lectures from MedImmune/Abbott, outside the submitted work. C. Gremmen has nothing to disclose. T. Halvorsen has nothing to disclose. J. Kamphuis has nothing to disclose. S. Kotecha has nothing to disclose. K. Rooney-Otero has nothing to disclose. S. Schulzke has nothing to disclose. A. Wilson has nothing to disclose. D. Rigau acts as ERS methodologist. R.L. Morgan has nothing to disclose. T. Tonia acts as ERS methodologist. C.C. Roehr has nothing to disclose. M.W. Pijnenburg has nothing to disclose.

Support statement: The participants of the project received funding for travel and meetings from the European Respiratory Society (number TF-2015-18). Funding information for this article has been deposited with the Crossref Funder Registry.

\section{References}

1 Stoll BJ, Hansen NI, Bell EF, et al. Trends in care practices, morbidity, and mortality of extremely preterm neonates, 1993-2012. JAMA 2015; 314: 1039-1051.

2 Johnson AH, Peacock JL, Greenough A, et al. High-frequency oscillatory ventilation for the prevention of chronic lung disease of prematurity. N Engl J Med 2002; 347: 633-642.

3 Chowdhury O, Wedderburn CJ, Duffy D, et al. CPAP review. Eur J Pediatr 2012; 171: 1441-1448.

4 Jacobsen T, Grønvall J, Petersen S, et al. "Minitouch" treatment of very low-birth-weight infants. Acta Paediatr 1993; 82: 934-938.

5 Miksch RM, Armbrust S, Pahnke J, et al. Outcome of very low birthweight infants after introducing a new standard regime with the early use of nasal CPAP. Eur J Pediatr 2008; 167: 909-916.

6 Narendran V, Donovan EF, Hoath SB, et al. Early bubble CPAP and outcomes in ELBW preterm infants. J Perinatol 2003; 23: 195-199.

7 Aly H, Milner JD, Patel K, et al. Does the experience with the use of nasal continuous positive airway pressure improve over time in extremely low birth weight infants? Pediatrics 2004; 114: 697-702.

8 De Klerk AM, De Klerk RK. Nasal continuous positive airway pressure and outcomes of preterm infants. J Paediatr Child Health 2001; 37: 161-167.

9 Wheeler K, Klingenberg C, McCallion N, et al. Volume-targeted versus pressure-limited ventilation in the neonate. Cochrane Database Syst Rev 2010; 1: CD003666. 
10 SUPPORT Study Group of the Eunice Kennedy Shriver NICHD Neonatal Research Network, Finer NN, Carlo WA, et al. Early CPAP versus surfactant in extremely preterm infants. N Engl J Med 2010; 362: 1970-1979.

11 Cools F, Askie LM, Offringa M, et al. Elective high-frequency oscillatory versus conventional ventilation in preterm infants: a systematic review and meta-analysis of individual patients' data. Lancet 2010; 375: 2082-2091.

12 Cools F, Offringa M, Askie LM. Elective high frequency oscillatory ventilation versus conventional ventilation for acute pulmonary dysfunction in preterm infants. Cochrane Database Syst Rev 2015; 3: CD000104.

13 Dunn MS, Kaempf J, de Klerk A, et al. Randomized trial comparing 3 approaches to the initial respiratory management of preterm neonates. Pediatrics 2011; 128: e1069-e1076.

14 Morley CJ, Davis PG, Doyle LW, et al. Nasal CPAP or intubation at birth for very preterm infants. N Engl J Med 2008; 358: 700-708.

15 Liggins GC, Howie RN. A controlled trial of antepartum glucocorticoid treatment for prevention of the respiratory distress syndrome in premature infants. Pediatrics 1972; 50: 515-525.

16 Taeusch HW Jr, Wong YL, Torday JS, et al. Maternal glucocorticoid treatment and reduction of risk from respiratory distress syndrome. J Reprod Med 1979; 23: 252-256.

17 Roberts D, Dalziel S. Antenatal corticosteroids for accelerating fetal lung maturation for women at risk of preterm birth. Cochrane Database Syst Rev 2006; 3: CD004454.

18 Murphy KE, Hannah ME, Willan AR, et al. Multiple courses of antenatal corticosteroids for preterm birth (MACS): a randomised controlled trial. Lancet 2008; 372: 2143-2151.

19 Sweet DG, Carnielli V, Greisen G, et al. European consensus guidelines on the management of respiratory distress syndrome - 2019 update. Neonatology 2019; 115: 432-450.

20 Banerjee S, Fernandez R, Fox GF, et al. Surfactant replacement therapy for respiratory distress syndrome in preterm infants: United Kingdom national consensus. Pediatr Res 2019; 86: 12-14.

21 Welsford M, Nishiyama C, Shortt C, et al. Room air for initiating term newborn resuscitation: a systematic review with meta-analysis. Pediatrics 2019; 143: e20181825.

22 Welsford M, Nishiyama C, Shortt C, et al. Initial oxygen use for preterm newborn resuscitation: a systematic review with meta-analysis. Pediatrics 2019; 143: e20181828.

23 Askie LM, Darlow BA, Finer N, et al. Association between oxygen saturation targeting and death or disability in extremely preterm infants in the Neonatal Oxygenation Prospective Meta-Analysis collaboration. JAMA 2018; 319: 2190-2201.

24 van Zanten HA, Pauws SC, Stenson BJ, et al. Effect of a smaller target range on the compliance in targeting and distribution of oxygen saturation in preterm infants. Arch Dis Child Fetal Neonatal Ed 2018; 103: F430-F435.

25 Schmidt B, Whyte RK, Asztalos EV, et al. Effects of targeting higher $v s$ lower arterial oxygen saturations on death or disability in extremely preterm infants: a randomized clinical trial. JAMA 2013; 309: 2111-2120.

26 Askie LM, Darlow BA, Davis PG, et al. Effects of targeting lower versus higher arterial oxygen saturations on death or disability in preterm infants. Cochrane Database Syst Rev 2017; 4: CD011190.

27 Jobe AJ. The new BPD: an arrest of lung development. Pediatr Res 1999; 46: 641-643.

28 Jobe AH, Bancalari E. Bronchopulmonary dysplasia. Am J Respir Crit Care Med 2001; 163: 1723-1729.

29 Eichenwald EC, Stark AR. Management and outcomes of very low birth weight. N Engl J Med 2008; 358: $1700-1711$.

30 Eber E, Zach MS. Long term sequelae of bronchopulmonary dysplasia (chronic lung disease of infancy). Thorax 2001; 56: 317-323.

31 Aukland SM, Rosendahl K, Owens CM, et al. Neonatal bronchopulmonary dysplasia predicts abnormal pulmonary HRCT scans in long-term survivors of extreme preterm birth. Thorax 2009; 64: 405-410.

32 Kotecha SJ, Edwards MO, Watkins WJ, et al. Effect of preterm birth on later $\mathrm{FEV}_{1}$ : a systematic review and meta-analysis. Thorax 2013; 68: 760-766.

33 Moschino L, Stocchero M, Filippone M, et al. Longitudinal assessment of lung function in survivors of bronchopulmonary dysplasia from birth to adulthood. The Padova BPD Study. Am J Respir Crit Care Med 2018; 198: 134-137.

34 Baraldi E, Filippone M. Chronic lung disease after premature birth. N Engl J Med 2007; 357: 1946-1955.

35 Hülskamp G, Lum S, Stocks J, et al. Association of prematurity, lung disease and body size with lung volume and ventilation inhomogeneity in unsedated neonates: a multicentre study. Thorax 2009; 64: 240-245.

36 Satrell E, Røksund O, Thorsen E, et al. Pulmonary gas transfer in children and adolescents born extremely preterm. Eur Respir J 2013; 42: 1536-1544.

37 Fawke J, Lum S, Kirkby J, et al. Lung function and respiratory symptoms at 11 years in children born extremely preterm: the EPICure study. Am J Respir Crit Care Med 2010; 182: 237-245.

38 Welsh L, Kirkby J, Lum S, et al. The EPICure study: maximal exercise and physical activity in school children born extremely preterm. Thorax 2010; 65: 165-172.

39 Simpson SJ, Turkovic L, Wilson AC, et al. Lung function trajectories throughout childhood in survivors of very preterm birth: a longitudinal cohort study. Lancet Child Adolesc Health 2018; 2: 350-359.

40 Cheong JLY, Doyle LW. An update on pulmonary and neurodevelopmental outcomes of bronchopulmonary dysplasia. Semin Perinatol 2018; 42: 478-484.

41 Smith VC, Zupancic JA, McCormick MC, et al. Rehospitalization in the first year of life among infants with bronchopulmonary dysplasia. J Pediatr 2004; 144: 799-803.

42 Allen J, Zwerdling R, Ehrenkranz R, et al. Statement on the care of the child with chronic lung disease of infancy and childhood. Am J Respir Crit Care Med 2003; 168: 356-396.

43 Rosenfeld M, Allen J, Arets BH, et al. An official American Thoracic Society workshop report: optimal lung function tests for monitoring cystic fibrosis, bronchopulmonary dysplasia, and recurrent wheezing in children less than 6 years of age. Ann Am Thorac Soc 2013; 10: S1-S11.

44 Guyatt GH, Oxman AD, Vist GE, et al. GRADE: an emerging consensus on rating quality of evidence and strength of recommendations. BMJ 2008; 336: 924-926.

45 Guyatt GH, Oxman AD, Kunz R, et al. GRADE guidelines: 2. Framing the question and deciding on important outcomes. J Clin Epidemiol 2011; 64: 395-400.

46 Andrews JC, Schünemann HJ, Oxman AD, et al. GRADE guidelines: 15. Going from evidence to recommendation determinants of a recommendation's direction and strength. J Clin Epidemiol 2013; 66: 726-735. 
47 Ronkainen E, Perhomaa M, Mattila L, et al. Structural pulmonary abnormalities still evident in schoolchildren with new bronchopulmonary dysplasia. Neonatology 2018; 113: 122-130.

48 Tonson la Tour A, Spadola L, Sayegh Y, et al. Chest CT in bronchopulmonary dysplasia: clinical and radiological correlations. Pediatr Pulmonol 2013; 48: 693-698.

49 Maconochie I, Greenough A, Yuksel B, et al. A chest radiograph scoring system to predict chronic oxygen dependency in low birth weight infants. Early Hum Dev 1991; 26: 37-43.

50 Mahut B, De Blic J, Emond S, et al. Chest computed tomography findings in bronchopulmonary dysplasia and correlation with lung function. Arch Dis Child Fetal Neonatal Ed 2007; 92: F459-F464.

51 Northway WH Jr, Rosan RC, Porter DY. Pulmonary disease following respirator therapy of hyaline-membrane disease. Bronchopulmonary dysplasia. N Engl J Med 1967; 276: 357-368.

52 Urs R, Kotecha S, Hall GL, et al. Persistent and progressive long-term lung disease in survivors of preterm birth. Paediatr Respir Rev 2018; 28: 87-94.

53 Higano NS, Spielberg DR, Fleck RJ, et al. Neonatal pulmonary magnetic resonance imaging of bronchopulmonary dysplasia predicts short-term clinical outcomes. Am J Respir Crit Care Med 2018; 198: 1302-1311.

54 Förster K, Ertl-Wagner B, Ehrhardt H, et al. Altered relaxation times in MRI indicate bronchopulmonary dysplasia. Thorax 2019; in press [doi: 10.1136/thoraxjnl-2018-212384].

55 Bentsen $\mathrm{MH}$, Markestad T, Øymar $\mathrm{K}$, et al. Lung function at term in extremely preterm-born infants: a regional prospective cohort study. BMJ Open 2017; 7: e016868.

56 Proietti E, Riedel T, Fuchs $\mathrm{O}$, et al. Can infant lung function predict respiratory morbidity during the first year of life in preterm infants? Eur Respir J 2014; 43: 1642-1651.

57 Vom Hove M, Prenzel F, Uhlig HH, et al. Pulmonary outcome in former preterm, very low birth weight children with bronchopulmonary dysplasia: a case-control follow-up at school age. J Pediatr 2014; 164: 40-45.

58 Broström EB, Thunqvist P, Adenfelt G, et al. Obstructive lung disease in children with mild to severe BPD. Respir Med 2010; 104: 362-370.

59 den Dekker HT, Sonnenschein-van der Voort AMM, de Jongste JC, et al. Early growth characteristics and the risk of reduced lung function and asthma: a meta-analysis of 25,000 children. J Allergy Clin Immunol 2016; 137: 1026-1035.

60 Zivanovic S, Peacock J, Alcazar-Paris M, et al. Late outcomes of a randomized trial of high-frequency oscillation in neonates. N Engl J Med 2014; 370: 1121-1130.

61 Gabriele C, Nieuwhof EM, Van Der Wiel EC, et al. Exhaled nitric oxide differentiates airway diseases in the first two years of life. Pediatr Res 2006; 60: 461-465.

62 Baraldi E, Bonetto G, Zacchello F, et al. Low exhaled nitric oxide in school-age children with bronchopulmonary dysplasia and airflow limitation. Am J Respir Crit Care Med 2005; 171: 68-72.

63 Filippone M, Bonetto G, Corradi M, et al. Evidence of unexpected oxidative stress in airways of adolescents born very pre-term. Eur Respir J 2012; 40: 1253-1259.

64 Teig N, Allali M, Rieger C, et al. Inflammatory markers in induced sputum of school children born before 32 completed weeks of gestation. J Pediatr 2012; 161: 1085-1090.

65 Latzin P, Roth S, Thamrin C, et al. Lung volume, breathing pattern and ventilation inhomogeneity in preterm and term infants. PLoS One 2009; 4: e4635.

66 Schulzke SM, Hall GL, Nathan EA, et al. Lung volume and ventilation inhomogeneity in preterm infants at 15-18 months corrected age. J Pediatr 2010; 156: 542-549.

67 Choukroun ML, Feghali H, Vautrat S, et al. Pulmonary outcome and its correlates in school-aged children born with a gestational age $\leqslant 32$ weeks. Respir Med 2013; 107: 1966-1976.

68 Sørensen JK, Buchvald F, Berg AK, et al. Ventilation inhomogeneity and NO and CO diffusing capacity in ex-premature school children. Respir Med 2018; 140: 94-100.

69 Gough A, Linden M, Spence D, et al. Impaired lung function and health status in adult survivors of bronchopulmonary dysplasia. Eur Respir J 2014; 43: 808-816.

70 Oostveen E, MacLeod D, Lorino H, et al. The forced oscillation technique in clinical practice: methodology, recommendations and future developments. Eur Respir J 2003; 22: 1026-1041.

71 Clouse BJ, Jadcherla SR, Slaughter JL. Systematic review of inhaled bronchodilator and corticosteroid therapies in infants with bronchopulmonary dysplasia: implications and future directions. PLoS One 2016; 11: e0148188.

72 Yuksel B, Greenough A. Randomised trial of inhaled steroids in preterm infants with respiratory symptoms at follow up. Thorax 1992; 47: 910-913.

73 Pelkonen AS, Hakulinen AL, Hallman M, et al. Effect of inhaled budesonide therapy on lung function in schoolchildren born preterm. Respir Med 2001; 95: 565-570.

74 Kao LC, Durand DJ, McCrea RC, et al. Randomized trial of long-term diuretic therapy for infants with oxygen-dependent bronchopulmonary dysplasia. J Pediatr 1994; 124: 772-781.

75 Askie LM, Henderson-Smart DJ, Irwig L, et al. Oxygen-saturation targets and outcomes in extremely preterm infants. N Engl J Med 2003; 349: 959-967.

76 Primhak R. Oxygen titration strategies in chronic neonatal lung disease. Paediatr Respir Rev 2010; 11: 154-157.

77 Hayes D Jr, Wilson KC, Krivchenia K, et al. Home oxygen therapy for children. An official American Thoracic Society clinical practice guideline. Am J Respir Crit Care Med 2019; 199: e5-e23.

78 Boost II United Kingdom Collaborative Group, Boost II Australia Collaborative Group, Boost II New Zealand Collaborative Group, et al. Oxygen saturation and outcomes in preterm infants. $N$ Engl J Med 2013; 368: 2094-2104.

79 Balfour-Lynn IM, Field DJ, Gringras P, et al. BTS guidelines for home oxygen in children. Thorax 2009; 64: Suppl. 2, ii1-ii26.

80 van der Graaf M, Rojer LA, Helbing W, et al. EXPRESS: Sildenafil for bronchopulmonary dysplasia and pulmonary hypertension: a meta-analysis. Pulm Circ 2019: 2045894019837875.

81 Mourani PM, Mandell EW, Meier M, et al. Early pulmonary vascular disease in preterm infants is associated with late respiratory outcomes in childhood. Am J Respir Crit Care Med 2019; 199: 1020-1027.

82 Mourani PM, Abman SH. Pulmonary vascular disease in bronchopulmonary dysplasia: pulmonary hypertension and beyond. Curr Opin Pediatr 2013; 25: 329-337.

83 Mourani PM, Sontag MK, Younoszai A, et al. Early pulmonary vascular disease in preterm infants at risk for bronchopulmonary dysplasia. Am J Respir Crit Care Med 2015; 191: 87-95. 
84 Ehrenkranz RA, Walsh MC, Vohr BR, et al. Validation of the National Institutes of Health consensus definition of bronchopulmonary dysplasia. Pediatrics 2005; 116: 1353-1360.

85 Walsh MC, Yao Q, Gettner P, et al. Impact of a physiologic definition on bronchopulmonary dysplasia rates. Pediatrics 2004; 114: 1305-1311.

86 Crump C, Sundquist J, Winkleby MA, et al. Preterm birth and risk of chronic kidney disease from childhood into mid-adulthood: national cohort study. BMJ 2019; 365: 11346.

87 Haraldsdottir K, Watson AM, Beshish AG, et al. Heart rate recovery after maximal exercise is impaired in healthy young adults born preterm. Eur J Appl Physiol 2019; 119: 857-866.

88 Goss KN, Beshish AG, Barton GP, et al. Early pulmonary vascular disease in young adults born preterm. Am J Respir Crit Care Med 2018; in press [doi: 10.1164/rccm.201710-2016OC].

89 Lewandowski AJ, Bradlow WM, Augustine D, et al. Right ventricular systolic dysfunction in young adults born preterm. Circulation 2013; 128: 713-720.

90 Naumburg E, Söderström L, Huber D, et al. Risk factors for pulmonary arterial hypertension in children and young adults. Pediatr Pulmonol 2017; 52: 636-641.

91 Ehrmann DE, Mourani PM, Abman SH, et al. Echocardiographic measurements of right ventricular mechanics in infants with bronchopulmonary dysplasia at 36 weeks postmenstrual age. J Pediatr 2018; 203: 210-217.

92 Higgins $\mathrm{RD}$, Jobe $\mathrm{AH}$, Koso-Thomas $\mathrm{M}$, et al. Bronchopulmonary dysplasia: executive summary of a workshop. J Pediatr 2018; 197: 300-308.

93 Shepherd EG, Knupp AM, Welty SE, et al. An interdisciplinary bronchopulmonary dysplasia program is associated with improved neurodevelopmental outcomes and fewer rehospitalizations. J Perinatol 2012; 32: 33-38.

94 van Mastrigt E, Kakar E, Ciet P, et al. Structural and functional ventilatory impairment in infants with severe bronchopulmonary dysplasia. Pediatr Pulmonol 2017; 52: 1029-1037.

95 Guyatt GH, Oxman AD, Kunz R, et al. Going from evidence to recommendations. BMJ 2008; 336: 1049-1051. 